\title{
The static three-quark potential from the Polyakov loop correlation function
}

\author{
Yoshiaki Koma*t \\ National Institute of Technology, Numazu College \\ E-mail: koma@numazu-ct.ac.jp \\ Miho Koma \\ Nihon University, College of International Relations \\ E-mail: koma.miho@nihon-u.ac.jp
}

\begin{abstract}
We present results of the static three-quark potential and the force in SU(3) lattice gauge theory at zero temperature extracted from the Polyakov loop correlation function (PLCF) by employing the multilevel algorithm. We investigate various cases that the three quarks are placed at the vertices of the equilateral, the isosceles, and the right triangles. We find that all the potential data, except for the cases such that the two of three quarks are very close to each other, can be explained by one curve as a function of the $Y$-distance defined by the minimum extent of lines connecting all three quarks via the Fermat point of the triangle. We also find that the three-quark force at long $Y$-distances is quantitatively the same as that of the quark-antiquark force.
\end{abstract}

The 32nd International Symposium on Lattice Field Theory

23-28 June, 2014

Columbia University New York, NY

\footnotetext{
* Speaker.

${ }^{\dagger}$ Y.K. is partially supported by JSPS KAKENHI Grant Number 24740176. The main calculation has been performed on the NEC SX8 at Research Center for Nuclear Physics (RCNP), Osaka University, Japan.
} 


\section{Introduction}

The static three-quark potential is one of the typical quantities in QCD reflecting its SU(3) gauge symmetry, which is relevant to heavy baryon spectroscopy, but its feature is less known compared to the quark-antiquark potential. Lattice QCD simulations offer nonperturbative computation of the potential, and so far, the three-quark potential has been investigated by several groups, where the "baryonic" Wilson loop as illustrated in Fig. 11(left) has been computed as an extension of the ordinary rectangular Wilson loop for the quark-antiquark potential [1, 2, 3, 4, 5, 6, 7].

All groups have obtained similar results to the quark-antiquark potential that the functional form of the potential is described by the Coulombic term at short distances and a linearly-rising confining term at long distances. However, they arrived at two different conclusions concerning the underlying structure of the potential at long distances. One is the $\Delta$-type structure [1, 2, 3, 4, 7, and the other is the $Y$-type structure [5, 6]. The idea of the $\Delta$-type structure originates from the fact that the three-quark potential can be described effectively by the sum of the three two-body potentials, which means that the $\Delta$-shaped color flux-tube is formed among the three quarks. On the other hand, the idea of the $Y$-type structure is from the fact that the three-quark potential is well-fitted to the linear function of the distance that minimizes the total extent of lines connecting the three quarks. This indicates that all color flux-tubes associated with the three quarks meet at a junction called the Fermat point, which signals the presence of genuine three-body interaction.

Recently, the present authors have shown that the static three-quark potential can be investigated from the Polyakov loop correlation function (PLCF) [8], composed of spatially separated three Polyakov loops as illustrated in Fig.1 (right), by employing the multilevel algorithm [10 at zero temperature. As discussed in Ref. [8], the topologically $Y$-shaped spatial Wilson lines with a junction in the baryonic Wilson loop may cause systematic effects to extract the ground state potential at long distances even if one uses smearing techniques [11], while there is no such a problem in the PLCF. In fact, we have compared our result of the equilateral-triangle-shaped three-quark potential from the PLCF with that from the baryonic Wilson loop [6] and found a distinct deviation at long distances [8].

In this paper, we extend our previous computation of the three-quark potential from the PLCF. By applying the multilevel algorithm extensively, we investigate the potential that the three quarks are placed at the vertices not only of the equilateral triangles but also of the isosceles and the right triangles, up to $3.1 \mathrm{fm}$ with the $\Delta$-distance and $1.8 \mathrm{fm}$ with the $Y$-distance. We then find a systematic behavior especially with the $Y$-distance such that all the potential data, except for the cases such that the two of three quarks are very close to each other, fall into one curve. We also compute the force among the three quarks with respect to the $\Delta$ - and $Y$-distances, and find that the long distance behavior of the force with the $Y$-distance is the same as that of the quark-antiquark system, indicating that they have the common string tension.

\section{The three-quark potential from the PLCF}

The three-quark potential is computed from the PLCF as follows. We consider SU(3) lattice gauge theory in four dimensions with the lattice volume $L^{3} \times T$ and the lattice spacing $a$, and impose periodic boundary conditions in all space-time directions. We first prepare the three-link 

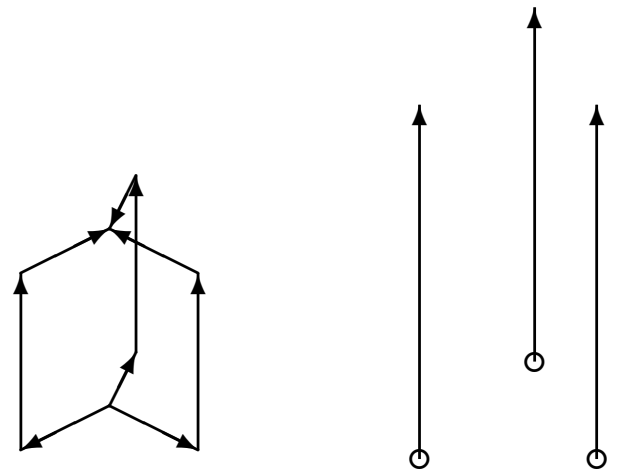

Figure 1: The baryonic Wilson loop (left) and the baryonic PLCF (right).

correlators, a tensor product of the time-like link variables $U_{4}(x)$ placed at $x=\left(x_{0}, \vec{x}_{1}\right),\left(x_{0}, \vec{x}_{2}\right)$ and $\left(x_{0}, \vec{x}_{3}\right)$ for $x_{0}=0, a, 2 a, \ldots, T-a$,

$$
\mathbb{T}\left(x_{0}, \vec{x}_{1,2,3}\right)_{\alpha \beta \gamma \delta \varepsilon \zeta} \equiv U_{4}\left(x_{0}, \vec{x}_{1}\right)_{\alpha \beta} U_{4}\left(x_{0}, \vec{x}_{2}\right)_{\gamma \delta} U_{4}\left(x_{0}, \vec{x}_{3}\right)_{\varepsilon \zeta},
$$

where Greek indices $\alpha, \beta, \gamma, \delta, \varepsilon, \zeta$ take the values from 1 to 3, respectively. Practically, a threelink correlator is a complex matrix with $9 \times 9 \times 9=729$ components. The multiplication law of the three-link correlators of the adjacent time slices at $x_{0}$ and $x_{0}+a$ is

$$
\left\{\mathbb{T}\left(x_{0}, \vec{x}_{1,2,3}\right) \mathbb{T}\left(x_{0}+a, \vec{x}_{1,2,3}\right)\right\}_{\alpha \beta \gamma \delta \varepsilon \zeta}=\mathbb{T}\left(x_{0}, \vec{x}_{1,2,3}\right)_{\alpha \lambda \gamma \rho \varepsilon \sigma} \mathbb{T}\left(x_{0}+a, \vec{x}_{1,2,3}\right)_{\lambda \beta \rho \delta \sigma \zeta},
$$

where repeated indices are to be summed over. The three-link correlator acts on the color state in the $\mathbf{3} \otimes \mathbf{3} \otimes \mathbf{3}$ representation of the SU(3) group $\left|n ; \vec{x}_{1,2,3}\right\rangle_{\alpha \beta \gamma}$, which is the eigenstate of the hamiltonian $\mathbb{H}$ defined by the transfer matrix in the temporal gauge, $\mathbb{T} \equiv e^{-\mathbb{H} a}$, and then satisfies $\mathbb{T}\left(x_{0}, \vec{x}_{1,2,3}\right)_{\alpha \lambda \beta \rho \gamma \sigma}\left|n ; \vec{x}_{1,2,3}\right\rangle_{\alpha \beta \gamma}=e^{-E_{n}\left(\vec{x}_{1,2,3}\right) a}\left|n ; \vec{x}_{1,2,3}\right\rangle_{\lambda \rho \sigma}$, where $n$ is the principal quantum number and $E_{n}\left(\vec{x}_{1,2,3}\right)$ corresponds to the energies. Note that $E_{n}\left(\vec{x}_{1,2,3}\right)$ are common to all color components of $\left|n ; \vec{x}_{1,2,3}\right\rangle_{\alpha \beta \gamma}$.

Using the three-link correlators, the operator for the baryonic PLCF is constructed as

$$
\operatorname{Tr} P\left(\vec{x}_{1}\right) \operatorname{Tr} P\left(\vec{x}_{2}\right) \operatorname{Tr} P\left(\vec{x}_{3}\right)=\left\{\mathbb{T}\left(0, \vec{x}_{1,2,3}\right) \mathbb{T}\left(a, \vec{x}_{1,2,3}\right) \cdots \mathbb{T}\left(T-a, \vec{x}_{1,2,3}\right)\right\}_{\alpha \alpha \gamma \gamma \varepsilon \varepsilon} .
$$

By inserting the complete set of eigenstates $1=\sum_{m=0}^{\infty}\left|m ; \vec{x}_{1,2,3}\right\rangle\left\langle m ; \vec{x}_{1,2,3}\right|$ at all time slices $x_{0}=$ $0, a, \ldots, T-a$, the expectation value is written as

$$
\left\langle\operatorname{Tr} P\left(\vec{x}_{1}\right) \operatorname{Tr} P\left(\vec{x}_{2}\right) \operatorname{Tr} P\left(\vec{x}_{3}\right)\right\rangle=\sum_{n=0}^{\infty} w_{n} e^{-E_{n}\left(\vec{x}_{1,2,3}\right) T},
$$

with $w_{0}=1$, which is guaranteed by construction. The ground state potential $V\left(\vec{x}_{1,2,3}\right) \equiv E_{0}\left(\vec{x}_{1,2,3}\right)$ is then extracted as

$$
V\left(\vec{x}_{1,2,3}\right)=-\frac{1}{T} \ln \left\langle\operatorname{Tr} P\left(\vec{x}_{1}\right) \operatorname{Tr} P\left(\vec{x}_{2}\right) \operatorname{Tr} P\left(\vec{x}_{3}\right)\right\rangle+O\left(\frac{1}{T} e^{-\left(E_{1}-E_{0}\right) T}\right),
$$

where the error terms of $O\left(\frac{1}{T} e^{-\left(E_{1}-E_{0}\right) T}\right)$ are already negligible at zero temperature. Note that if one uses the Wilson loop, the weight factor $w_{n}$ is dependent both on the location of the three quarks and the junction of the spatial Wilson lines so that the value of $w_{0}$ is unknown a priori. Moreover, 
the error terms become of $O\left(\frac{1}{t} e^{-\left(E_{1}-E_{0}\right) t}\right)$ for the temporal extent of the Wilson loop $t$, which are not easily suppressed for larger spatial separation of quarks, since $t$ cannot be large practically. There is also limitation such as $t<T / 2$ due to the periodic boundary condition. In this sense, the extraction of the potential from the PLCF is theoretically cleaner than that from the Wilson loop, once the PLCF is computed accurately.

One problem is that the expectation values of the PLCF are extremely small at long distances, which are immediately obscured by the statistical noise within the ordinary simulations. Using the multilevel algorithm [9, 10], however, it is possible to overcome this problem. The idea is to compute a desired correlation function, which may have an extremely small expectation value, from the product of relatively large sublattice averages of its components, where the sublattices are defined by dividing the lattice volume into several layers along the time direction. During the computation of the sublattice averages, the spatial links at the sublattice boundaries are fixed. The computation of the correlation function in this way is supported by the transfer matrix formalism and is regarded as the hierarchical functional integral method.

As an example, let us consider a simple case that the lattice volume is divided into two sublattices $\left(N_{\text {sub }}=2\right)$ at the time slice $x_{0}=0$ and $x_{0}=T / 2$. The number of the time slices in a sublattice is $N_{\text {tsl }}=T /\left(a N_{\text {sub }}\right)$. We then compute the averages of $\left\{\mathbb{T}\left(0, \vec{x}_{1,2,3}\right) \cdots \mathbb{T}\left(T / 2-a, \vec{x}_{1,2,3}\right)\right\}_{\alpha \beta \gamma \delta \varepsilon \zeta}$ and $\left\{\mathbb{T}\left(T / 2, \vec{x}_{1,2,3}\right) \cdots \mathbb{T}\left(T-a, \vec{x}_{1,2,3}\right)\right\}_{\alpha \beta \gamma \delta \varepsilon \zeta}$ and construct the operator by

$$
\begin{aligned}
& \operatorname{Tr} P\left(\vec{x}_{1}\right) \operatorname{Tr} P\left(\vec{x}_{2}\right) \operatorname{Tr} P\left(\vec{x}_{3}\right) \\
& =\left[\mathbb{T}\left(0, \vec{x}_{1,2,3}\right) \cdots \mathbb{T}\left(\frac{T}{2}-a, \vec{x}_{1,2,3}\right)\right]_{\alpha \lambda \gamma \rho \varepsilon \sigma}\left[\mathbb{T}\left(\frac{T}{2}, \vec{x}_{1,2,3}\right) \cdots \mathbb{T}\left(T-a, \vec{x}_{1,2,3}\right)\right]_{\lambda \alpha \rho \gamma \sigma \varepsilon},
\end{aligned}
$$

where $[\cdots]$ represents taking the sublattice average. Note that fixing the spatial links at the sublattice boundaries correspond to inserting two normalized fixed sources $\left|\phi_{1}\right\rangle_{\alpha \beta \gamma}=\sum_{n=0}^{\infty} a_{n}\left|n ; \vec{x}_{1,2,3}\right\rangle_{\alpha \beta \gamma}$ and $\left|\phi_{2}\right\rangle_{\alpha \beta \gamma}=\sum_{m=0}^{\infty} b_{m}\left|m ; \vec{x}_{1,2,3}\right\rangle_{\alpha \beta \gamma}$ at $x_{0}=0$ and $x_{0}=T / 2$, respectively, where $a_{n}$ and $b_{m}$ are unknown a priori but satisfy $\sum_{n=0}^{\infty}\left|a_{n}\right|^{2}=\sum_{n=0}^{\infty}\left|b_{n}\right|^{2}=1$. Then, Eq. (2.6) becomes

$$
\begin{aligned}
\operatorname{Tr} P\left(\vec{x}_{1}\right) \operatorname{Tr} P\left(\vec{x}_{2}\right) \operatorname{Tr} P\left(\vec{x}_{3}\right) & =\operatorname{Tr}\left[\left\langle\phi_{1}\left|\mathbb{T}(0) \cdots \mathbb{T}\left(\frac{T}{2}-a\right)\right| \phi_{2}\right\rangle\right]\left[\left\langle\phi_{2}\left|\mathbb{T}\left(\frac{T}{2}\right) \cdots \mathbb{T}(T-a)\right| \phi_{1}\right\rangle\right] \\
& =\sum_{\alpha \lambda} \sum_{\gamma \rho} \sum_{\varepsilon \sigma}\left(\sum_{n=0}^{\infty} a_{n}^{*} b_{n} e^{-E_{n}\left(\vec{x}_{1,2,3}\right)(T / 2)} \cdot \sum_{m=0}^{\infty} b_{m}^{*} a_{m} e^{-E_{m}\left(\vec{x}_{1,2,3}\right)(T / 2)}\right)
\end{aligned}
$$

If we take the average for different fixed sources at $x_{0}=0$ and $x_{0}=T / 2$ of other independent gauge configurations, we obtain $\left\langle\operatorname{Tr} P\left(\vec{x}_{1}\right) \operatorname{Tr} P\left(\vec{x}_{2}\right) \operatorname{Tr} P\left(\vec{x}_{3}\right)\right\rangle$ as in Eq. 2.4D, since inserting the fixed sources finally reduces to inserting the complete set.

At this point, it is worth noting that if $T / 2$ is large enough so that the terms of $O\left(e^{-\left(E_{1}-E_{0}\right)(T / 2)}\right)$ are negligible, which is usually the case at zero temperature, Eq. (2.7) further reduces to

$$
\operatorname{Tr} P\left(\vec{x}_{1}\right) \operatorname{Tr} P\left(\vec{x}_{2}\right) \operatorname{Tr} P\left(\vec{x}_{3}\right)=\sum_{\alpha \lambda} \sum_{\gamma \rho} \sum_{\varepsilon \sigma} \underbrace{\left|a_{0}\right|^{2}\left|b_{0}\right|^{2} e^{-E_{0}\left(\vec{x}_{1,2,3}\right) T}}_{\text {independent of } \alpha, \lambda, \gamma, \rho, \varepsilon, \sigma}=3^{6}\left|a_{0}\right|^{2}\left|b_{0}\right|^{2} e^{-E_{0}\left(\vec{x}_{1,2,3}\right) T} .
$$

In this case, the ground state potential $E_{0}\left(\vec{x}_{1,2,3}\right)$ can be extracted even from "one" configuration, where the energy $E_{0}\left(\vec{x}_{1,2,3}\right)$ is independent of the color components of the intermediate states.

In general, the lattice volume can be divided into $N_{\text {sub }}$ sublattices for $N_{\text {sub }} \geq 2$, if the terms of $O\left(e^{-\left(E_{1}-E_{0}\right)\left(a N_{\text {ts }}\right)}\right)$ are still negligible. If this is not the case, the sublattice averaging must be 

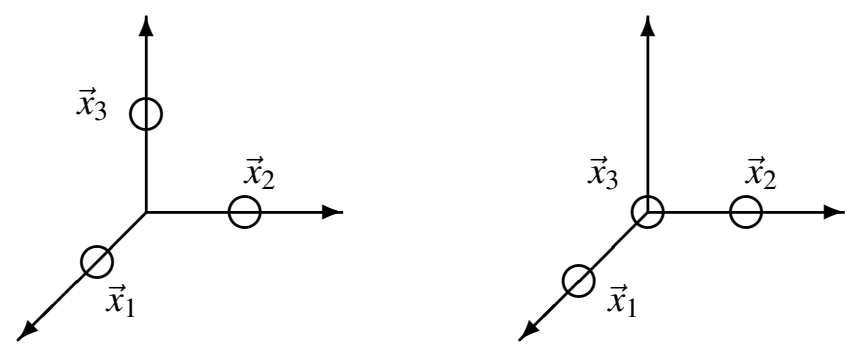

Figure 2: The spatial locations where the three Polyakov loops are placed (the three-quark location): the equilateral- and the isosceles-triangle cases (left) and the right-triangle case (right).

carried out up to higher $k$-levels until the terms of $O\left(e^{-\left(E_{1}-E_{0}\right)\left(a k N_{\text {tsl }}\right)}\right)$ become negligible. Otherwise the ground state energy cannot be extracted due to contamination from the excited states. In our experience with the SU(3) Wilson gauge action, there is a critical minimal length of $a N_{\text {tsl }}$ to obtain the ground state energy. We find the value $a N_{\text {tsl }} \simeq 0.38 \mathrm{fm}$ [12, 13, 14].

\section{Numerical results}

We carried out simulations using the standard Wilson gauge action in $\mathrm{SU}(3)$ lattice gauge theory at $\beta=6.00$ on the $24^{4}$ lattice with the multilevel algorithm. The lattice spacing is $a=$ $0.093[\mathrm{fm}]$, determined by the Sommer scale $r_{0}=0.50[\mathrm{fm}]$ [15]. One Monte Carlo update consisted of 1 heatbath and 5 over-relaxation steps. The number of sublattice is $N_{\text {sub }}=6$, which corresponds to $N_{\mathrm{tsl}}=4$.

We investigated the three-quark potential and force for the cases that the three quarks are placed at the vertices of the equilateral, the isosceles, and the right triangles, which are then plotted as functions of two typical distances with the $\Delta$ - and $Y$-types, $R_{\Delta}$ and $R_{Y}$, respectively, where $R_{\Delta}$ is the sum of the extent of lines between the two of three quarks, while $R_{Y}$ is the minimum extent of lines connecting the three quarks via the Fermat point of the triangle. The spatial location of the three quarks is shown schematically in Fig. 2 We distinguish the location of the three quarks (the shape of the triangle) with the notation like "rrr", "r11", "r10", etc. For example, "rrr" means $\vec{x}_{1}=(r, 0,0), \vec{x}_{2}=(0, r, 0), \vec{x}_{3}=(0,0, r)$, where $r$ varies simultaneously, which is the equilateral triangle case. " $\mathrm{r} 11$ " is $\vec{x}_{1}=(r, 0,0), \vec{x}_{2}=(0,1,0), \vec{x}_{3}=(0,1,0)$, which is the isosceles triangle case. "r10" is $\vec{x}_{1}=(r, 0,0), \vec{x}_{2}=(0,1,0), \vec{x}_{3}=(0,0,0)$, which is the right triangle case, and so on.

In Fig. 3, we plot a typical history of the PLCF of "one" configuration for the "rrr" case as a function of the number of internal sublattice updates $N_{\text {iupd }}$ (left) and the corresponding potential at $N_{\text {iupd }}=500000$ as a function of $R_{Y}$ (right), which is compared to the average of the potential from nine independent configurations at the same $N_{\text {iupd }}$. We find that the result from one configuration coincides with the average, which means that it is possible to compute the expectation value even from one configuration by tuning the parameters of the multilevel algorithm as explained in the previous section.

In Fig. 4 we then show results of the three-quark potentials for various triangle cases as functions of $R_{\Delta}$ (left) and $R_{Y}$ (right), respectively, from the same "one" configuration at $N_{\text {iupd }}=$ 500000 used in Fig. 3. The shapes of the potentials look quite similar to each other, but we find a systematic behavior especially in the $Y$-type plot since all the potential data fall into one curve, 

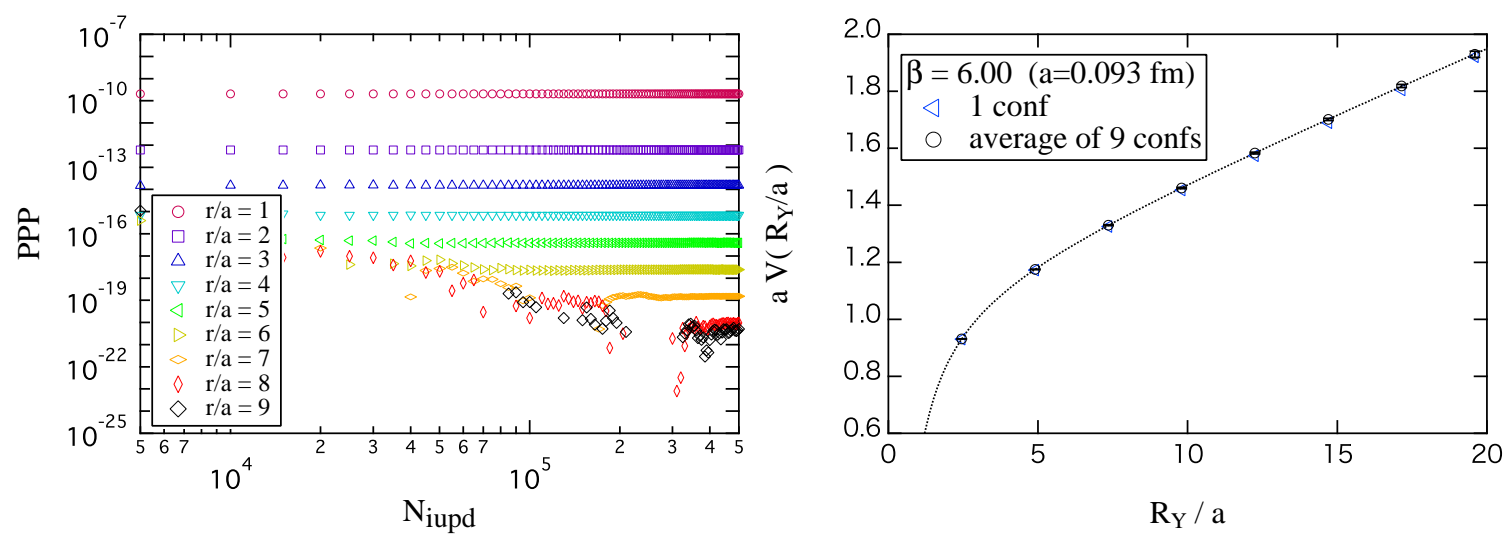

Figure 3: A typical history of the PLCF of "one" configuration for the equilateral triangle case (left) and the corresponding potential at $N_{\text {iupd }}=500000$ (right), which is compared to the average of the potential from nine independent configurations at the same $N_{\text {iupd }}$.
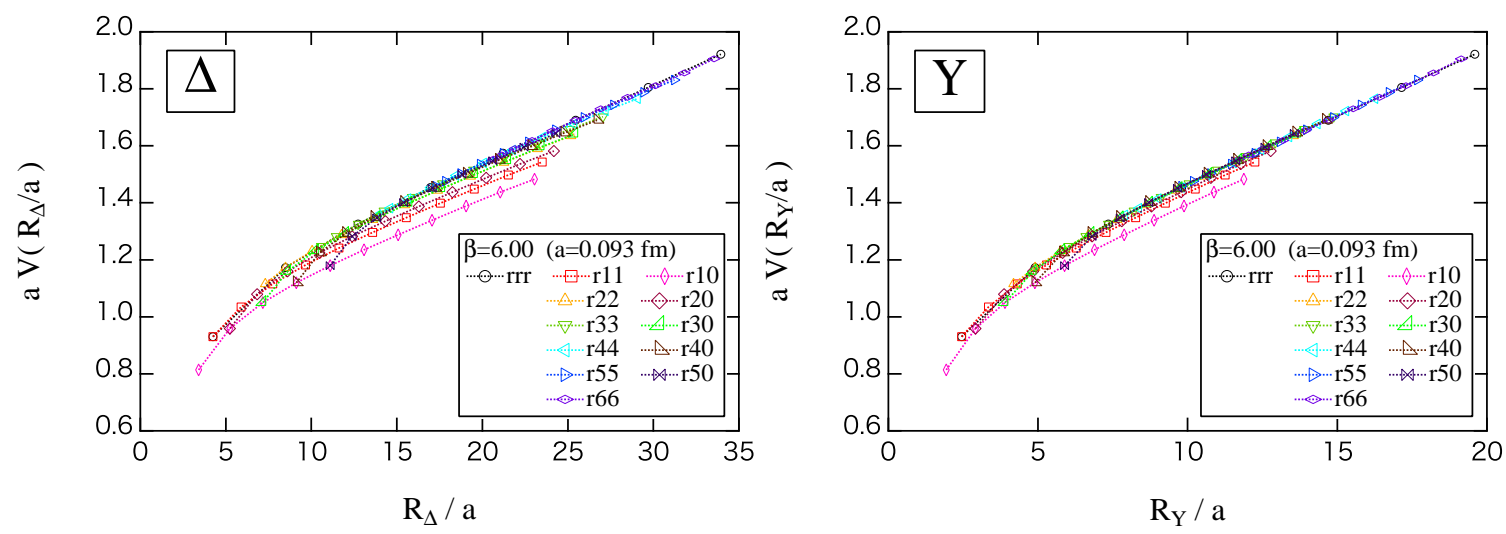

Figure 4: The three-quark potentials as a function of the $\Delta$-distance (left) and of the $Y$-distance (right). The meaning of the notation such as "rrr", "r11", "r10", etc. are explained in the text.

except for the cases such that the two of three quarks are very close to each other, which may be due to short distance effects.

In Fig. 5, we show results of the three-quark forces with the $\Delta$-type $\frac{d V\left(R_{\Delta}\right)}{d R_{\Delta}}$ (left), and the $Y$-type $\frac{d V\left(R_{Y}\right)}{d R_{Y}}$ (right), respectively. The behaviors of the forces are again similar to each other as expected from that of the potentials, but we find that the convergence property at long distances of the $Y$-type plot is better than that of the $\Delta$-type plot. Moreover, the "string tension" from the $Y$-type plot is consistent with that of the quark-antiquark potential $\sigma a^{2}=0.0468$ at $\beta=6.00$ [13]. We observe the universal feature of the string tension in the $Y$-type plot as pointed out in Ref. [5, 6] without fitting procedures.

\section{Summary}

We have computed the static three-quark potential and the force in SU(3) lattice gauge theory at zero temperature from the Polyakov loop correlation function (PLCF) by employing the multilevel algorithm. Compared to the use of the Wilson loop, there is no artificial junction of the spatial 

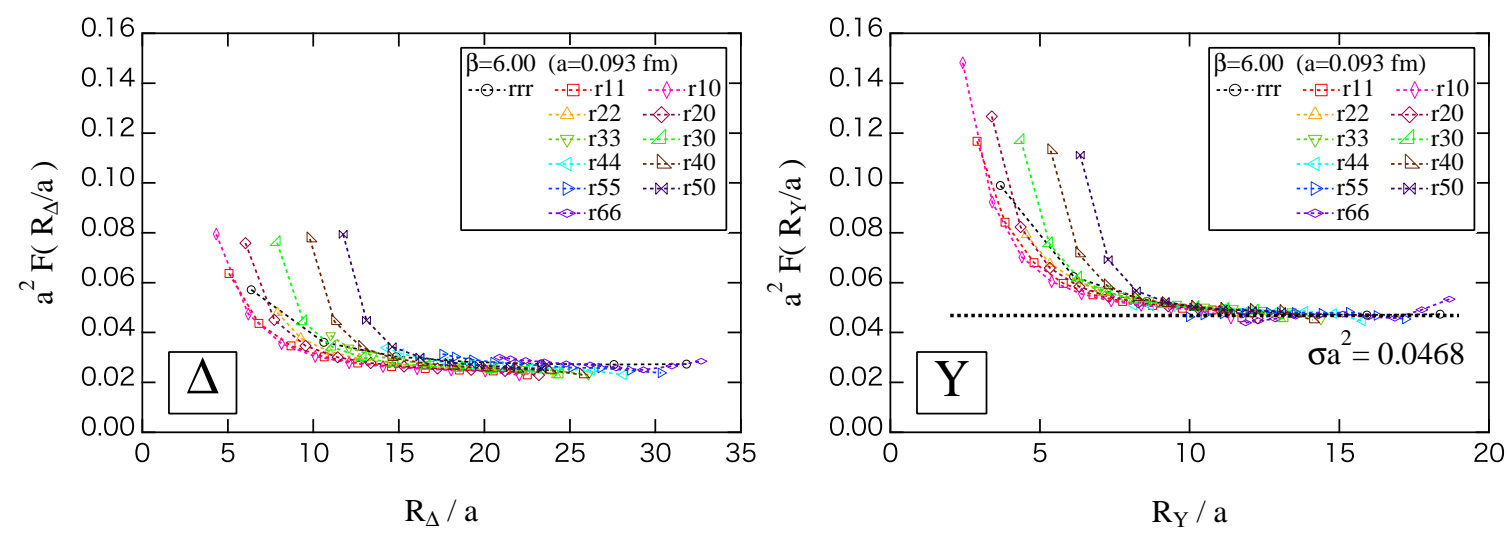

Figure 5: The three-quark force as a function of the $\Delta$-distance (left) and of the $Y$-distance (right). The meaning of the notation such as "rrr", "r11", "r10", etc. are the same as that in Fig. 4.

Wilson lines for the three-quark source and sink in the PLCF, which is useful to obtain the ground state potential with less systematic effects.

We have investigated various cases that the three quarks are placed at the vertices of the equilateral, the isosceles, and the right triangles. We have found that all the potential data, except for the cases such that the two of three quarks are very close to each other, can be explained by one curve as a function of the $Y$-distance defined by the minimum extent of lines connecting all three quarks via the Fermat point of the triangle. We have also computed the three-quark force with respect to the $\Delta$ - and $Y$-distances, and have found that its behavior at long $Y$-distances is quantitatively the same as that of the quark-antiquark force.

The three-quark potential for other cases such as the obtuse triangles can also be investigated along this line, which will be presented in our forthcoming paper.

\section{References}

[1] R. Sommer and J. Wosiek, Phys.Lett. B149 (1984) 497.

[2] R. Sommer and J. Wosiek, Nucl.Phys. B267 (1986) 531.

[3] H. Thacker, E. Eichten, and J. Sexton, Nucl.Phys.Proc.Suppl. 4 (1988) 234.

[4] G. S. Bali, Phys.Rept. 343 (2001) 1-136.

[5] T. T. Takahashi, H. Matsufuru, Y. Nemoto, and H. Suganuma, Phys.Rev.Lett. 86 (2001) 18-21.

[6] T. T. Takahashi, H. Suganuma, Y. Nemoto, and H. Matsufuru, Phys.Rev. D65 (2002) 114509.

[7] C. Alexandrou, Ph. de Forcrand, and A. Tsapalis, Phys.Rev. D65 (2002) 054503.

[8] Y. Koma and M. Koma, PoS LATTICE2013 (2013) 469.

[9] M. Lüscher and P. Weisz, JHEP 09 (2001) 010.

[10] M. Lüscher and P. Weisz, JHEP 07 (2002) 049.

[11] APE Collaboration, M. Albanese et al., Phys.Lett. B192 (1987) 163-169.

[12] Y. Koma, M. Koma, and H. Wittig, Phys.Rev.Lett. 97 (2006) 122003.

[13] Y. Koma and M. Koma, Nucl.Phys. B769 (2007) 79-107.

[14] Y. Koma and M. Koma, PoS LAT2009 (2009) 122.

[15] S. Necco and R. Sommer, Nucl. Phys. B622 (2002) 328-346. 